\title{
COMPLEX ZEBRA PATTERNS IN SOLAR RADIO EMISSION AND GENERATION MECHANISMS
}

\author{
G. P. Chernov* and V. V. Fomichev*
}

\begin{abstract}
Several complex uncommon zebra patterns in meter/ decimeter and microwave range are shown. It is difficult to interpret them as the regular harmonics within the framework of known models, e.g. based on the double plasma resonance (DPR) mechanism. Specifically, for this reason, only in the last 5 years about 10 works devoted to an improvement of the DPR mechanism were published, and 5 new models were proposed. We propose a new advanced model of ZP based on the explosive instability in the system of the weakly-relativistic mono-velocity beam of protons - the strongly nonisothermic plasma.
\end{abstract}

\section{Introduction}

A disputable discussion about nature of zebra pattern (ZP) in the type IV radio bursts continues in the literature. The explanation to this is connected with the new observations of the uncommon forms of ZP. According to all works, which analyze mechanism based on the DPR, we must observe $\mathrm{ZP}$ in all continuous bursts. But observations suggest otherwise: ZP appears not in all bursts and usually in the pulsation regime. Nevertheless, it is sometimes asserted that mechanism on DPR explains all basic properties of ZP, and it is indicated on the error of many authors in the calculations of the increments of plasma waves at the upper hybrid frequency $\omega_{U H}$ [Zlotnik, 2009]: it is not possible to enlarge calculations for the regions adjacent with the hybrid band, since the dispersion relation

$$
\omega^{2} \simeq \omega_{P e}^{2}+\omega_{B e}^{2}+3 k_{\perp}^{2} v_{T}^{2},
$$

is correct only inside the hybrid band. Here, $\omega_{P e}$ and $\omega_{B e}$ are plasma and cyclotron frequencies, $k_{\perp}$ is wave number perpendicular to the magnetic field and $v_{T}$ is electron thermal velocity. However, in a whole series of works it is noted that the contribution of the term, connected with the harmonic in the hybrid band, considerably exceeds contribution

* IZMIRAN, Russian Academy of Sciences, Troitsk, 142190, Moscow Region, Russia 
from those harmonics adjacent from above, especially for the slightly non-perpendicular propagation in the relativistic examination [Robinson, 1988]. New observations and incompleteness of many known mechanisms stimulate for the new developments.

Here, it is shown that in the system the weakly-relativistic mono-velocity beam of protons - the strongly nonisothermic plasma the slow beam mode can possess negative energy and the explosive instability with interaction of slow and rapid beam modes and ionic sound is developed. As a result of the weak spatial dispersion the generation of ionic sound is accompanied by the cascade process of coalescence, and the stabilization of explosive instability occurs. ZP is formed by scattering of fast protons on the ion-acoustic harmonics. The effectiveness of new mechanism in comparison with the previously discussed hypotheses is explained. The assumption about the presence of the beams of fast protons in the nonisothermic plasma is natural for any large flare.

\section{Generation Mechanisms}

The understanding of the nature of fine structures of a radio emission is one of the major criteria of check of radio emission mechanisms. Discussion about an origin of the zebra-structure proceeds more than 30 years. The mechanism based on a double plasma resonance [Zheleznykov and Zlotnik, 1975] is more often discussed:

$$
\omega_{U H}=\left(\omega_{P e}^{2}+\omega_{B e}^{2}\right)^{1 / 2}=s \omega_{B e}
$$

Fiber-bursts differ from stripes of zebra-structure by only constant frequency drift in the dynamic spectrum. However it is possible to consider as the standard mechanism of radiation of fibers the interaction of plasma waves with whistlers, raised simultaneously with plasma waves on the upper hybrid frequency by fast electrons with loss-cone velocity distribution: $l+w \rightarrow t$ [Kuijpers, 1975]. The entire magnetic trap can be divided into intermittent layers of whistler amplification and absorption.

In some phenomena ZP- stripes smoothly transform into fibers and back that testifies to the uniform nature of these two types of stripes in emission and absorption [Chernov, 2006].

In the recent small critical review of Zlotnik [2009], the advantages of the DPR model and the main failures of the model with whistlers are refined. The statement that the theory based on whistlers is able to explain only a single stripe (e.g., a fiber burst) was made in Zlotnik [2009] without the corresponding ideas of whistler excitation and propagation in the solar corona.

According to Gladd [1983], the growth rate of whistlers for relativistic energies of fast particles decreases slightly if the full relativistic dispersion is used. Then, Tsang [1984] specified calculations of relativistic growth rates of whistlers with the loss-cone distribution function. The brightness temperature of electromagnetic radiation $T_{b}$ in result of coupling of Langmuir and whistler waves depends mainly on effective temperature of whistlers $T_{w}$ due to $T_{l} \gg T_{w}$ and in the Manley-Rowe relation for $T_{b}$ the second term in the 
denominator $\omega_{w} T_{l}$ should be $\gg \omega_{l} T_{w}$ [Kuijpers, 1975], (formula (32) in page 66). Thus, our conclusion, that the entire magnetic trap can be divided into intermittent layers of whistler amplification and absorption remains valid for a broad energy range of fast particles.

In Zlotnik [2009] the main matter which is ignored is that the model involves quasilinear interactions of whistlers with fast particles, allowing one to explain all the fine effects of the ZP dynamics, mainly the superfine structure of ZP stripes, the frequency splitting, and the oscillating frequency drift of the stripes which occurs synchronously with the spatial drift of radio sources.

\section{New Observation}

In a meter wave band the complex combination of fibers and a zebra-structure is sometimes observed. In Fig. 1 periodic narrow-band fibers (similar to ropes), shipped in a developed zebra-structure in the meter wave band on IZMIRAN spectra are shown.
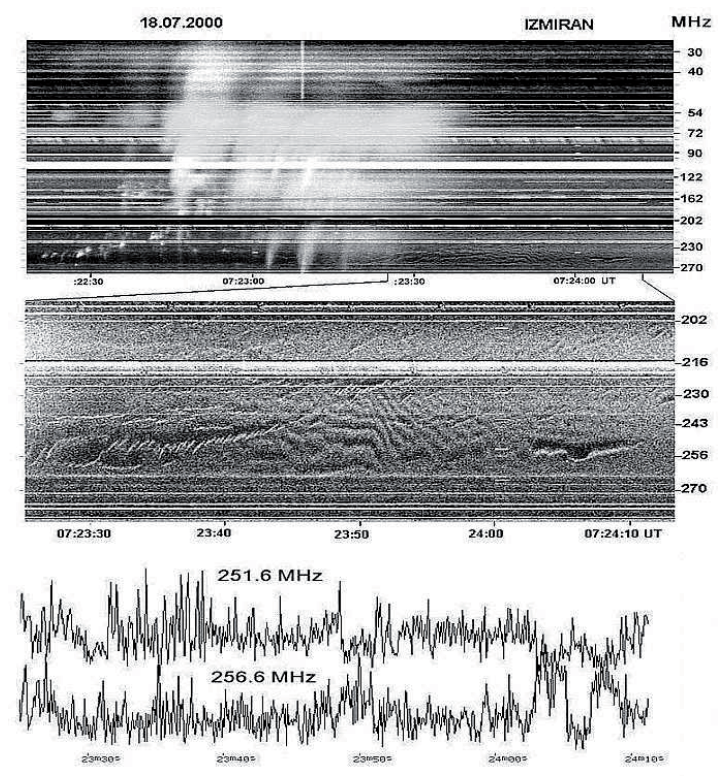

Figure 1: IZMIRAN dynamic spectrum (25-270 MHz) for the beginning of the July 18, 2000 event (upper panel). The middle panel shows a magnified fragment of the rope of fibers in the developed ZP. The intensity profiles are shown below on the same time scale with a magnified spectrum; they show the modulation depth of the emission during the rope (251.6- $\mathrm{MHz}$ profile) and a single zigzag fiber (256.6- $\mathrm{MHz}$ profile). 
In two events 2004 July 24 and 2004 November 3 (Fig. 2) the large-scale ZP consisted of small-scale fiber bursts. The appearance of such an uncommon fine structure is connected with the following special features of the plasma wave excitation in the radio source: both whistler and plasma wave instabilities are too weak at the very beginning of the events (the continuum was absent), and the fine structure is almost invisible. Then, whistlers generated directly at DPR levels "highlight" the radio emission only from these levels due to their interaction with plasma waves.

In a microwave range the unusual fine structure consisting of millisecond spikes in absorption was found. It was observed with Chinese spectrometer in a range $2.6-3.8 \mathrm{GHz}$ (Huajrou station, Beijing) in last large flare (X3.4/4B) of 23rd cycle at December, 13th, 2006. The analysis of the optical data allows to believe that the continuum radiation can be generated in a magnetic trap by loss-cone instability of fast electrons. A unique fine structure was observed: spikes in absorption formed dark ZP stripes against the absorptive type III-like bursts. The spikes in absorption can appear in accordance with the well known mechanism of absorptive bursts (according to Zaitsev and Stepanov [1975]). The additional injection of fast particles filled the loss-cone (breaking the loss-cone distribution), and the generation of the continuum was quenched at these moments, which was evidenced by the formation of bursts in absorption.

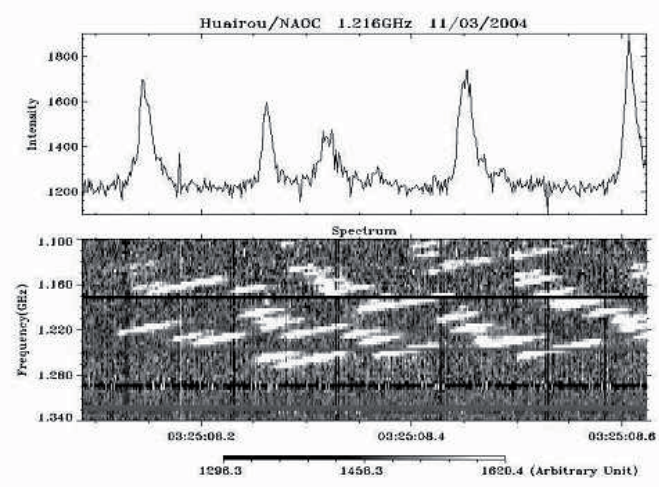

Figure 2: The magnified dynamic spectrum (bottom) and the time profile at $1.216 \mathrm{GHz}$ (top) of the fiber structure on 2004 November 3.

The maximum absorptive effect occurred at the DPR levels. The parameters of millisecond spikes are determined by small dimensions of the particle beams and local scale heights in the radio source (as Chen and Yan [2008] showed).

In Fig. 4 the entire emission of a short event 29 May 2003 consists of spikes. The spikes form several fibers with different frequency drift and several fragments of zebra pattern with different scales of the frequency separation between stripes.

We have shown some phenomena with very complex fine structures. Since all known models still have deficiencies, the attempts to create new theories continue. 


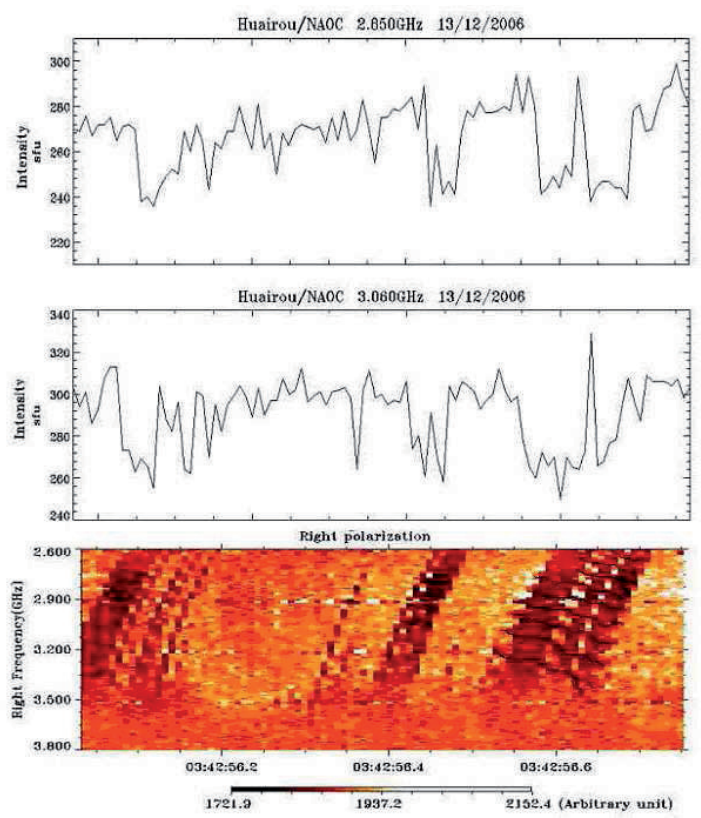

Figure 3: Enlarged spectrum and time profiles at two fixed frequencies (2.85 and $3.06 \mathrm{GHz})$ of $\approx 0.7 \mathrm{~s}$ duration showing the modulations during the type III-like bursts in absorption with a ZP. The absorptive ZP-like stripes are marked by thin black lines (from Chernov et al. [2010]).

\section{Other Mechanisms}

In practically all models the discussion deals with regular ZP. Problems appear with the interpretation of the frequently observed uncommon stripes of a ZP. To overcome difficulties arising in different models, a new ZP theory based on the emission of auroral choruses (magnetospheric bursts) via the escape of the $\mathrm{Z}$ mode captured by regular plasma density inhomogeneities was recently proposed [LaBelle et al., 2003]. This theory, however, fails to explain the high intensity of radiation emitted by separate incoherent sources. In addition, the theory imposes some stringent conditions, such as the presence of a largeamplitude ion-acoustic wave.

The formation of transparency and opacity bands during the propagation of radio waves through regular coronal inhomogeneities is the most natural and promising mechanism [Laptuhov and Chernov, 2009]. Several aspects of this mechanism were considered in Laptukhov and Chernov [2006]; Barta and Karlicky [2006]; Ledenev et al. [2006]. It explains all main parameters of regular ZP. The dynamics of ZP stripes (variations in the frequency drift, stripe breaks, etc.) can be associated with the propagation of inhomogeneities, their evolution, and disappearance. Inhomogeneities are always present in the solar corona, however direct evidences of the existence of inhomogeneities with the 
scales of several meters in the corona are absent, although ion-sound waves could serve this purpose.

\section{The New Alternative Mechanism of the ZP due to Develop- ment of Explosive Instability in the System Beam-plasma}

Observations of ZPs during powerful flares make it possible to assume that the particle acceleration to relativistic velocities and excitation of different wave modes occurs in the radio source. Therefore, probably, other possible interactions of waves and particles should be taken into account. For example, in Fomichev and Fainshtein [1981] the decay instability of whistlers to the harmonics of the ion sound, which have weak spatial dispersion and small damping at frequencies much less than the ion Langmuir frequency $\omega_{\text {oi }}$, was proposed. In Fomichev et al. [2009] an alternative mechanism of ZPs is discussed, due to the development of explosive instability in the system, which is a weakly relativistic beam with nonisothermic plasma.

The quasi-hydrodynamic approximation was utilized for describing the interaction of the particles of the beam and plasma [Fainshtein and Chernova, 1996; Ginzburg, 1967]. Linearizing the hydrodynamic equations for the processes of $\sim \exp (i \omega t-i k x)$, a dispersion equation for the system of flow-plasma was obtained. With speed of the ions of beam $V_{0} \gg c_{s}$ (the ion-sound velocity) the approximate dispersion equations were obtained:

$$
\begin{gathered}
\omega_{1} \equiv \Omega \approx c_{s} k_{1} \equiv c_{s} m q ; \\
\omega_{3,2}-k_{3,2} V_{0} \approx \mp \omega_{0 s}+\delta ; \frac{\delta}{\omega_{0 s}} \ll 1 .
\end{gathered}
$$

Equation (3) describes the ion-acoustic wave (energy positive), and (4) - slow ( $\omega_{3}$, negative energy) and rapid ( $\omega_{2}$, positive energy) beam waves. The number $m>0$ - is selected from the escape condition of radio emission from the corona. It is easy to determine that for the slow beam wave $\left(\omega_{3}, k_{3}\right)$, the fast beam wave $\left(\omega_{2}, k_{2}\right)$ and the sound $(\Omega, q)$ the conditions of synchronism are satisfied [Tsytovich, 1970]. From taking the conditions of synchronism (3), (14) into account we will obtain:

$$
m q \approx 2 \omega_{0 s} V_{0}^{-1}
$$

Since the sound has weak dispersion, the cascade process is possible:

$$
m q+m q \rightarrow 2 m q+m q \rightarrow 3 m q+m q \ldots m n q .
$$

Then, after decomposing nonlinear terms up to the quadratic terms and after using the standard procedure in the weak turbulence [Weiland and Wilhelmsson, 1977; Tsytovich, 1970], the shortened equations for the complex amplitudes of coupling modes are obtained: beam modes $a_{j}(j=1,2)$ and ion sound $b_{k}(k=1,2, \ldots m n)$. The analysis of interaction coefficients showed that the systems of such equations describe the stabilized "explosion" [Fainshtein, 1976]. It is also shown that the increment of the growth of ion sound in 


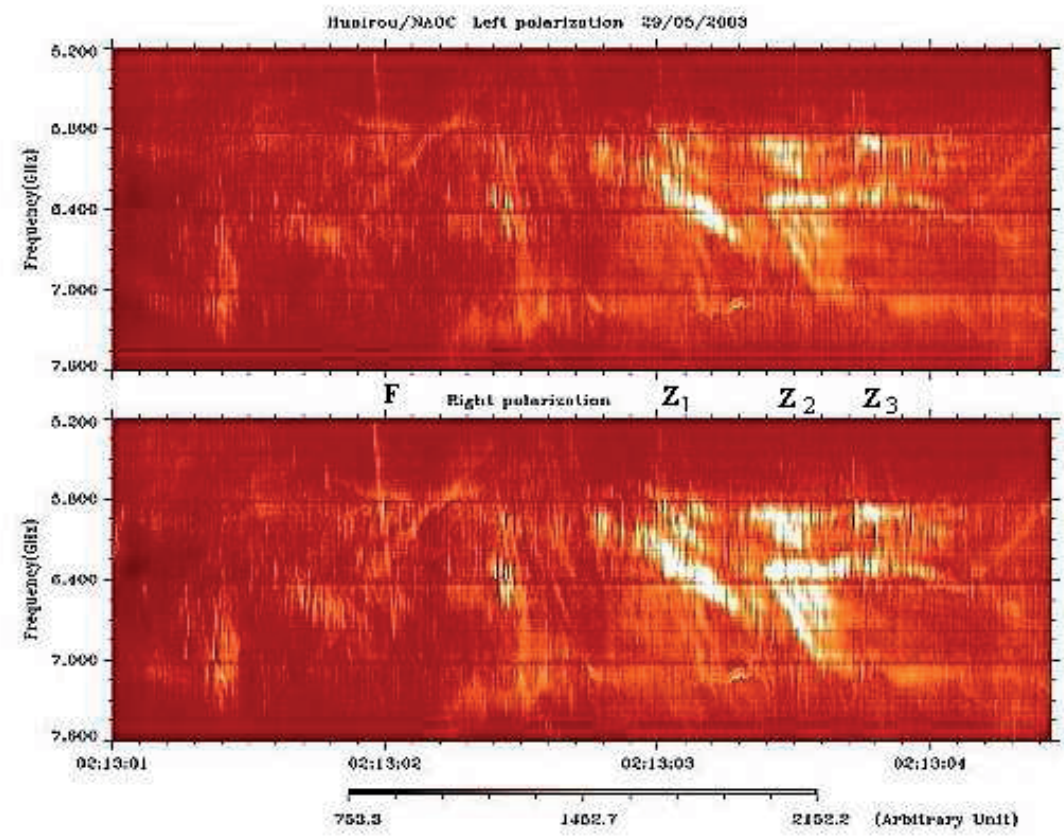

Figure 4: Complex fine structures with ZP in the 29 May 2003 event; the entire radio emission consists of millisecond spikes. The spikes form the fibers (noted between spectra in $L$ and $R$ polarization channels by letter $F$ ). Three fragments of $Z P$ with the different parameters are noted by symbols Z1, Z2, Z3.

this case considerably exceeds the values obtained in Fomichev and Fainshtein [1981]. Therefore the mechanism in question occurs much more effectively. The generable sound is scattered over the fast protons, which move with a speed of $V \sim V_{0} \sim 10^{10} \mathrm{cms}^{-1}$ and, according to the mechanism described in Fomichev and Faishtein [1981], the radiation from the source frequency is $\omega_{t} \approx m q n V$, and frequency separation between the stripes is $\delta \omega^{t}=m q V$. Taking into account equation (5) and selected parameters $\left(N_{0} \sim 5\right.$. $10^{9} \mathrm{~cm}^{-3}, N_{s} \cdot N_{0}^{-1} \sim 10^{-3}$, the constant magnetic field $\sim 30 \mathrm{G}$ ) we obtain for the emission frequency of $\geq 634 \mathrm{MHz}$ the value of coefficient $\mathrm{m}=15,\left(7 \cdot 10^{2} \leq \mathrm{mn} \ll 15 \cdot 10^{3}\right)$ and the frequency separation between the adjacent stripes $\delta \omega^{t} \approx 15 \mathrm{MHz}$. The obtained value of $\delta \omega^{t}$ corresponds to the observed frequency separation in the decimeter wave band [Chernov, 2006]. In the given above estimations the wavelength of the ion sound $\sim 100 \mathrm{~m}$, the initial frequency $\sim 1.0 \mathrm{kHz}$ and the cyclic frequency of the slow beam wave $\omega_{3} \sim 7 \cdot 10^{2} \omega_{0 i}$ (that correspond to $\sim 10 \mathrm{GHz}$ ).

The explosive instability appears in the nonequilibrium system, where there are waves of negative energy, moreover, in the resonance triplet the wave of the highest frequency of 
$\omega_{3}$ must possess by negative energy, and two lowest waves $\left(\omega_{1,2}\right)$ have positive energy.

\section{Conclusion}

New models don't yet allow to explain all new observant data in details. The rope-like fibers can be explained in terms of the model of fiber bursts [Chernov, 2008]. The connection of fibers with the developed zebra pattern (Fig.1) is shown within the framework of a unified approach to the formation theory of stripes in emission and absorption in the model on whistlers [Chernov, 2006]. The combined action of the DPR model and the mechanism with whistlers explains small-scale fibers which form a large-scale ZP (Fig. 2). If all the radiation consists of spikes (Fig.4), then the coalescence of plasma waves with the ion-acoustic waves (in a pulsating regime of their interaction with the whistlers) can be the most probable mechanism of their generation [Chernov et al., 2003]. The propagating ion-sound waves could be the most probable heterogeneities, on which the radio emission can be scattered with the formation of ZP (through the windows of transparency as eigenmodes of a resonator) [Barta and Karlicky, 2006; Laptuhov and Chernov, 2009]. The mechanism of scattering of fast protons on ion-sound harmonics in explosive instability looks as very uncommon. It could explain a regular ZP in large flares where the fast protons always exist, and the presence of nonisothermic plasma is completely feasible in the shock wave fronts.

Acknowledgements. G.P.C. appreciates the support of the Chinese Academy of Sciences and NSF of China (with Prof. Yan Yihua) as well as the Russian Foundation of Basic Research (RFBR), grant Nos. 08-02-00270 and 11-02-91151.

\section{References}

Barta, M. and M. Karlicky, Interference patterns in solar radio spectra: high-resolution structural analysis of the corona, Astron. Astrophys., 450, 359-364, 2006.

Breizman, B. N., in Problems in Plasma Theory, edited by B. B. Kadomtsev, Energoizdat, Moscow, 15, 55-145, 1987.

Chen, B., and Y. Yan, Short-lived absorptive type III- like microwave bursts as a signature of fragmented electron injections, Astrophys. J., 689, 1412-1420, 2008.

Chernov, G. P., Solar radio bursts with drifting stripes in emission and absorption, Space Sci. Rev., 127, 195-326, 2006.

Chernov, G. P., Unusual Stripes in Emission and Absorption in Solar Radio Bursts: Ropes of Fibers in the Meter Wave Band, Astron. Lett., 34, 486-499, 2008.

Chernov, G. P., Recent results of zebra patterns in solar radio bursts, Res. Astron. Astrophys., 10, 9, 821-866, 2010.

Chernov, G.P., Y. Yan and Q. J. Fu, A superfine structure in solar microwave bursts, Astron. Astrophys., 406, 1071-1081, 2003. 
Gladd, N. T., The whistler instability at relativistic energies, Phys. Fluids, 26, 974-982, 1983.

Fainshtein, S. M., Zhurn. Eksper. Teoret. Fiz., 71, 1021, 1976.

Fainstein, S. M., and E. A. Chernova, Zhurn. Eksper. Teoret. Fiz., 109, 821, 1996.

Fomichev, V.V., and S. M. Fainshtein, On a possible mechanism of zebra pattern generation in solar radio emission, Solar Phys., 71, 385, 1981.

Fomichev, V. V., S. M. Fainshtein, and G.P. Chernov, A Possible Interpretation of the Zebra Pattern in Solar Radiation, Plasma Phys. Rep., 35, 1032-1035, 2009.

Ginzburg, V. L., Propagation of Electromagnetic Waves in Plasma, Nauka, Moscow, 1967.

Kuznetsov, A. A., and Yu. T. Tsap, Loss-Cone Instability and Formation of Zebra Patterns in Type IV Solar Radio Bursts, Solar Phys., 241, 127-143, 2007.

Kuijpers, J., Collective wave-particle interactions in solar type IV radio source, Ph.D. thesis, Utrecht University, 1975.

LaBelle J., R. A. Treumann, P. H. Yoon, and M. Karlicky, A model of zebra emission in solar type IV radio bursts, Astrophys. J., 593, 1195-1207, 2003.

Laptuhov, A. I., and G. P. Chernov, New Mechanism for the Formation of Discrete Stripes in the Solar Radio Spectrum, Plasma Phys. Rep., 32, 866-871, 2006.

Laptuhov, A. I., and G. P. Chernov, Concerning Mechanisms for the Zebra Pattern Formation, in the Solar Radio Emission, Plasma Phys. Rep., 35, 160-168, 2009.

Ledenev, V.G., Y. Yan, and Q. Fu, Interference mechanism of "zebra-pattern" formation in solar radio emission, Solar Phys., 233, 129-138, 2006.

Robinson, P. A., Conditions for the validity of unmagnetized-plasma theory in describing weakly magnetized plasmas, Phys. Fluids, 31, 525-534, 1988.

Stepanov, A. V., and Y.T. Tsap, The energetic-electron spectra and hard X-ray radiation of solar flares, Astron. Rep., 43, 838-848, 1999.

Tsang, K. T., Electron-cyclotron maser and whistler instabilities in a relativistic electron plasma with loss cone distribution, Phys. Fluids, 27, 1659-1664, 1984.

Tsytovich, V. N., Nonlinear Effects in Plasma, Plenum Press, New York, 1970.

Weiland, J., and H. Wilhelmsson, Coherent Non-linear Interaction of Waves in Plasma, Pergamon Press, New York, 1977.

Winglee, R. M., and G.A. Dulk, The electron-cyclotron maser instability as a source of plasma radiation, Astrophys. J., 307, 808-819, 1986.

Zaitsev, V.V., and A.V. Stepanov, On the origin of drift absorption bursts, Astron. Astrophys., 45, 135-140, 1975.

Zheleznyakov, V.V., and E. Ya. Zlotnik, Cyclotron wave instability in the corona and origin of solar radio emission with fine structure, Solar Phys., 43, 431-451, 1975. 
Zlotnik, E. Ya., Origin of zebra pattern in type IV solar radio emission, Cent. Eur. Astrophys. Bull., 33, 281-298, 2009.

Zlotnik, E. Ya., and E. M. Sher, Toward a theory of the double plasma resonance in the solar corona, Radiophys. Quant. Electr., 52, 88-99, 2009.

Zlotnik, E. Ya., V. V. Zaitsev, H. Aurass, G. Mann, and A. Hofmann, Solar type IV burst spectral fine structures - Part II - Source model, Astron. Astrophys., 410, 10111027, 2003. 